\title{
Common Variant Associations with Fragile X Syndrome
}

James J Crowley $\mathrm{PhD}^{1{ }^{*}}$, Jin Szatkiewicz $\mathrm{PhD}^{1^{*}}$, Anna K Kähler PhD ${ }^{2 *}$, Paola Giusti-Rodriguez PhD ${ }^{1}$, NaEshia Ancalade PharmD ${ }^{1}$, Jessica K Booker PhD ${ }^{3}$, Jennifer L Carr MT MB ${ }^{3}$, Greg E Crawford PhD ${ }^{4}$, Molly Losh PhD ${ }^{5}$, Craig A Stockmeier PhD ${ }^{6}$, Annette K Taylor PhD ${ }^{7}$, Joseph Piven MD ${ }^{8,9+}$, Patrick F Sullivan MD FRANZCP ${ }^{1,2,8+}$

Department of Genetics, University of North Carolina at Chapel Hill, NC, USA

2 Department of Medical Epidemiology and Biostatistics, Karolinska Institutet, Stockholm, Sweden

3 Department of Pathology and Laboratory Medicine, University of North Carolina at Chapel Hill, NC, USA

4 Department of Pediatrics, Duke University, Durham, NC, USA

5 Department of Communication Sciences, Northwestern University, Evanston, IL, USA

6 University of Mississippi Medical Center, Jackson, MS, USA

7 Kimball Genetics, Denver, CO, USA

8 Department of Psychiatry, University of North Carolina at Chapel Hill, NC, USA

9 Carolina Institute for Developmental Disabilities, University of North Carolina at Chapel Hill, NC, USA

* These authors contributed equally. + co-corresponding authors.

Correspond with Drs Sullivan and Piven. Sullivan: Department of Genetics, CB\#7264, 5097 Genomic Medicine, University of North Carolina, Chapel Hill, NC, 27599-7264, USA, pfsulliv@med.unc.edu. Piven: Department of Psychiatry, CB\#7160, University of North Carolina, Chapel Hill, NC, 27599-7160, USA, joe piven@med.unc.edu.

\section{Abstract}

Fragile $X$ syndrome is a common cause of intellectual disability. It is usually caused by a de novo mutation which often occur on multiple haplotypes and should not be detectible using genome-wide association (GWA). We conducted GWA 89 male FXS cases and 266 male controls, and detected multiple genome-wide significant signals near FMR1 (odds ratio=8.10, $P=2.5 \times 10^{-10}$ ). These findings withstood robust attempts at falsification. Fine-mapping did not serve to narrow the interval (minimum $P=1.13 \times 10^{-14}$ ), and functional genomic integration (including $5 \mathrm{C}$ data we generated for this region) did not provide a mechanistic hypothesis. Controls carrying a risk haplotype had significantly longer and more variable FMR1 CGG repeats than controls with the protective haplotype $(P=4.75 \times 10$ $\left.{ }^{5}\right)$ which may predispose toward increases in CGG number to the pre-mutation range over many generations. This is a salutary reminder of the complexity of even "simple" monogenetic disorders.

\section{Introduction}

Fragile X syndrome (FXS) (1) is a common cause of intellectual disability $(0.25-1 / 1,000$ male births) (2, 3). It is characterized by intellectual disability, autistic behavior, hyperactivity, anxiety, and 
pleomorphic physical abnormalities (e.g., tall stature, macroorchidism). (4) FXS is caused by CGG expansion in the 5' UTR of the chromosome X gene FMR1 in most cases. (5-7) Full FXS mutations are characterized by expansion of the FMR1 5' UTR CGG repeat to $\geq 200$ copies with pre-mutations in the 55-200 copy range. (8)

FMR1 5' UTR CGG expansions generally arise as de novo mutations when mutable pre-mutations expand to full mutations during oogenesis. Although the probability of de novo mutations can be influenced by local DNA features, detection of de novo events using linkage disequilibrium would be unexpected for high-penetrance single gene disorders. $(9,10)$ This implies that genome-wide association (GWA) of FXS cases versus controls should not detect the FRM1 region as a susceptibility locus for FXS. As part of a study of FXS and autism, we conducted a case-control GWAS for FXS.

\section{Materials and Methods}

Subjects. Males with a genetically-confirmed diagnosis of FXS were recruited from volunteer registries (URLS). All available medical records were reviewed, and any features suggestive of a complex or atypical presentation led to exclusion. Controls were males from the Genes and Blood Clotting Study (GABC) in dbGaP (URLs, accession phs000304.v1.p1). GABC participants were male university students who volunteered for a study of the genetics of hemostasis and who had no acute or chronic illnesses. Additional male comparison subjects were from the Swedish Schizophrenia Study ( $N=3,525,46.4 \%$ cases). (11) As there is no evidence for association with schizophrenia in the FMR1 region, $(11,12)$ cases and controls were combined. We also used male HapMap3 founders from northwestern Europe and Tuscany (CEU and TSI, N=101). (13) All procedures were approved by Institutional Review Boards, and written informed consent was obtained from the parents/legal guardians of FXS cases and from control subjects.

Genetic assays. Table 1 summarizes the samples and assays used in this study. FMR1: the number of CGG repeats in the 5'UTR of FMR1 was determined in 89 FXS cases with a validated diagnostic assay (Kimball Genetics, Denver, CO). (14-16) To understand the internal structure of FMR1 CGG repeats and to place these on common haplotypes, we used AmplideX FMR1 PCR kits (Asuragen, Inc; Austin, TX; catalog \#49402) to quantify FMR1 5'UTR repeat sizes and to count AGG interruptions. GWAS: FXS cases and GABC controls were genotyped with Illumina HumanOmni1-Quad arrays, and genotypes were called using predefined clusters using GenomeStudio. Quality control was performed using PLINK. (17) SNPs were excluded for missingness $>0.03$, minor allele frequency (MAF) $<0.01$, deviation from Hardy-Weinberg expectations in controls $\left(P<1 \times 10^{-6}\right)$, SNP missingness differences between cases and controls $(P<0.05)$, or if a SNP probe did not map uniquely to the human genome. Subjects were excluded for missingness $>0.05$, excessive autosomal homozygosity or heterozygosity, 
or relatedness ( $\hat{\pi}>0.2$ based on LD pruned autosomal SNPS). One FXS case was genotyped in duplicate with 0.99998 concordance, and a CEPH sample previously assayed with the same array had 0.99981 concordance. TaqMan: rs2197706 and rs5905149 genotypes were verified with TaqMan Assays (catalog \#4351379 and \#4351379, Applied Biosystems, Carlsbad, CA). A SNP from Gerhardt et al. (18) (rs45631657) was genotyped with a custom TaqMan assay. Sequenom: we designed two massARRAY iPLEX (San Diego, CA) genotyping panels for common variant fine-mapping. SNPs were selected from GWAS results, haplotype analyses, and common variation databases, and then pruned using TAGGER. (19) All assays used genomic DNA isolated from peripheral blood. The genome reference was GRCh37/UCSC hg19.

Statistical analysis. Case-control comparisons were performed using PLINK (17) using logistic regression under an additive model with three ancestry principal components as covariates.

\section{Results}

We conducted GWA analyses for 750K SNPs in 89 male FXS cases and 266 male controls (Table S1). All FXS cases had full FMR1 mutations (>200 for the 5'UTR CGG repeat), and this was verified with a second assay ( 82 FXS cases with sufficient DNA). We assessed ancestry using principal components analysis (20) on LD-pruned autosomal SNPs (Figure S1). All controls and $90 \%$ of cases were of predominant European ancestry (we retained nine cases of mixed ancestry given the small number of cases). Logistic regression analyses identified five SNPs that met genome-wide significance with odds ratios $>5$ (Table 2, Figures 1a-b). These SNPs were in a $66 \mathrm{~kb}$ interval from chrX:146.85-146.92 $\mathrm{Mb}$ located $75 \mathrm{~kb} 5^{\prime}$ of the nearest gene, FMR1. Repeating the logistic regression conditioning on the most strongly associated SNP (rs2197706) markedly attenuated significance in the FMR1 region suggesting the presence of a single association signal.

Given that FXS usually results from de novo mutations, strong associations with common variation are unexpected. Indeed, the strongest association ( $r$ 2197706, odds ratio=8.10, $P=2.5 \times 10^{-10}$ ) is among the top dichotomous trait associations in the NHGRI/EBI GWAS catalog (21) (URLs). We therefore evaluated alternative explanations for these findings. First, given the marked allele frequency differences in cases and controls, re-genotyping rs5905149 and rs2197706 with TaqMan assays showed perfect agreement with Illumina array genotypes, and served to exclude allele assignment errors. Second, the allele frequencies in cases and controls were similar genome-wide except for SNPs 5' of FMR1 (Figure S2). Given our use of controls genotyped independently from cases, it is important to note that the control allele frequencies for the significant SNPs in the FMR1 region were similar to those from two external samples (Table 2 ). Third, genome-wide $P$-values conformed closely to the null expectation (mean $P$-value $=0.500$ over $750 K$ SNPs, Figure $1 a$ ), findings inconsistent 
with uncontrolled bias. Fourth, exclusion of nine cases with mixed ancestry had little impact on the results (Table 2). Fifth, a trivial explanation for these findings is if cases were cryptically related via a recent shared ancestor; however, case-case pairs were slightly less related on average than controlcontrol pairs (Figure S3). Cases and controls had similar proportions of autosomal homozygous SNPS as well as the number and size of autosomal runs of homozygosity (no comparisons were significantly different and cases had lower means in each instance). Sixth, asymptotic $P$-values can be inaccurate in small samples, but Fisher's exact test and permutation procedures yielded similar significance levels. Thus, we could identify no plausible alternative explanation for our findings.

In a fine-mapping experiment, we genotyped 32 SNPs (chrX:146844358-147013704, the association region extending into FMR1) in an expanded set of 97 FXS cases and 467 male controls (from a different study than for the initial GWAS). We included rs45631657which was reported to inactivate an important replication origin. (18) Variable numbers of SNPs overlapped with independent genotypes on the same subjects, and we observed $100 \%$ agreement (data not shown). Nine SNPS exceeded genome-wide significance (Table S2). All five SNPs in Table 2 replicated with consistent odd ratios and greater significance ( $P$-values ranging from $4 \times 10^{-12}$ to $\left.7 \times 10^{-14}\right)$, and four other SNPS reached genome-wide significance ( $r$ s4824231, $P=1.13 \times 10^{-14}$; rs25705, $P=5.74 \times 10^{-9} ;$ rs45631657, $P=5.20 \times 10^{-12}$; and $\left.\mathrm{rs} 112146098, P=6.60 \times 10^{-9}\right)$. Repeating the logistic regression conditioning on rs2197706 or rs4824231 markedly attenuated significance in the FMR1 region suggesting the presence of a single association signal. Thus, we continued to observe a broad region of significance.

Figure $1 \mathrm{c}$ depicts the $128 \mathrm{~kb}$ association region, from $141 \mathrm{~kb}$ to $13 \mathrm{~kb} \mathrm{5}$ of $F M R 1$. The association region includes the FMR1 promoter CGG repeat. Table 3 shows haplotypes from the genome-wide significant SNPs. The most common haplotype was strongly protective, and there were two risk haplotypes. FRM1 CGG analysis was available on 81 controls. Controls with the risk haplotype had significantly longer CGG repeats than controls with the protective haplotype (median 15 and interquartile range $13-22$ versus median 10 and interquartile range $\left.10-10, F_{1,79}=18.3, P=4.75 \times 10^{-5}\right)$. There was greater variability in CGG number in controls with the risk that the protective haplotype (standard deviation 2.4 vs 6.8). Around $40 \%$ of cases had additional phenotype measures (e.g., Vineland Adaptive Behavior Scale and the Social Responsiveness Scale) and there were no significant differences between cases with the risk or protective haplotypes (data not shown).

We next evaluated possible functions of the association region using functional genomic data (Figure 1c). Using RNA-seq data from human dorsolateral prefrontal cortex (DLPFC) in nine SCZ cases and nine controls along with prefrontal cortex (PFC) from nine fetuses and three neural progenitor cell lines, we saw that FMR1 (but not the antisense transcript, FMR1-AS1) was robustly expressed. There 
was no evidence of substantial gene expression or an unannotated feature in the association region $5^{\prime}$ to FMR1. The expression of FMR1 in DLPFC is associated with a common genetic variant but the associated SNP is far outside the region. (22) We evaluated DNA-DNA interactions using 5C (chromosome conformation capture-carbon copy) from five human fetal prefrontal cortex samples for a $1.26 \mathrm{Mb}$ region overlapping FMR1 and the association region (Figure S5). FMR1 promoters had evidence of DNA-DNA interactions with the association region and with other parts of FMR1. A microRNA gene cluster around $700 \mathrm{~kb} 5^{\prime}$ to $F M R 1$ had more substantial DNA-DNA interactions (Figure S5) but had no genetic association signal. We identified open chromatin using ATAC-seq (human DLPFC in nine SCZ cases and nine controls along with PFC from nine fetuses). The SNPS associated with FXS were not notable for open chromatin or key ChIP-seq marks.

\section{Discussion}

GWA of FXS cases versus controls identified an unusually strong association with the FMR1 region. The largest association (odds ratio $=8.10, P=2.5 \times 10^{-10}$ ) is among the top dichotomous trait associations in the NHGRI/EBI GWAS catalog (21) (URLs), and generally exceeded only by rare adverse drug reactions. Given the small sample size ( 89 FXS cases and 266 male controls), it is notable that the association survived robust attempts at falsification.

In some respects, our identification of the causal locus for FXS - a rare, single-gene disorder - in an outbred population using a linkage disequilibrium-based approach like GWA is unexpected. GWA in case-control samples can detect rare causal genes in special circumstances that do not apply here (e.g., when cases inherit a causal mutation from a relatively recent common ancestor $(23,24)$ or if multiple rare mutations yield an aggregate signal detectible by GWA (25)). De novo mutations in particular may be invisible to GWA: although de novo mutational processes can be influenced by local genomic context, replication timing, and genotypes at other loci, $(9,10,26-28)$ these effects are generally not deterministic, and most de novo mutations occur on different haplotypes.

With the exception of unusual exonic mutations, FXS is caused by a de novo mutational event in the expansion of a pre-mutation to a full mutation during oogenesis. (5-7) However, the local genomic context of FMR1 de novo promoter mutations is influential. (29-31) There is substantial evidence that this region is detectible via linkage disequilibrium in case-control studies using a few microsatellite markers. (32) Indeed, a 1992 paper (33) reported a FXS case versus control haplotype difference as " $P<0.001$ " but the $P$-value actually reached genome-wide significance $\left(P \sim 9 \times 10^{-9}\right)$. The association of common variation upstream of FMR1 with FXS has strong replication evidence in the literature: this is unquestionably a true association. 
Fine-mapping of the interval and integration with a number of types of functional genomic data did not narrow the region or yield a mechanistic hypothesis. A lack of early fetal data limits this conclusion. It is possible that a population genetic mechanism is at work: the risk haplotype is present in $18 \%$ of European-ancestry controls, and tends to carry a greater and more variable number of CGG repeats which may predispose toward increases in CGG number to the pre-mutation range over many generations. A similar mechanism has been reported for Huntington's disease. (34) This is a salutary reminder of the complexity of even "simple" monogenetic disorders.

\section{Conflicts of Interest}

PFS is a scientific advisor for Pfizer, Inc. and received an honorarium from F. Hoffmann-La Roche AG.

\section{Acknowledgements}

This project was funded by an Autism Speaks (URLs) award to PFS. PFS gratefully acknowledges support from the Swedish Research Council (Vetenskapsrådet, award D0886501). We are indebted to Dr Mark Daly for discussions regarding the results, and to Dr Job Dekker and his group for assistance with the $5 \mathrm{C}$ work. For the human postmortem samples, the authors acknowledge the Cuyahoga County Medical Examiner's Office and the families of the deceased. They also note contributions of Drs. James Overholser and George Jurjus and of Lesa Dieter in the retrospective psychiatric assessments, and Dr. Grazyna Rajkowska and Gouri Mahajan in sample preparation - this work was supported by NIH/NIGMS COBRE Center for Psychiatric Neuroscience (P30 GM103328).

URLS

Fragile X Research Registry, https://www.fragilexregistry.org. dbGaP, http://www.ncbi.nlm.nih.gov/gap. NHGRI GWAS catalog, https://www.ebi.ac.uk/gwas. Autism Speaks, http://www.autismspeaks.org 


\section{References}

1. Bhakar AL, Dolen G, Bear MF. The pathophysiology of fragile $X$ (and what it teaches us about synapses). Annu Rev Neurosci. 2012;35:417-43.

2. Turner G, Webb T, Wake S, Robinson H. Prevalence of fragile X syndrome. Am J Med Genet. 1996;64(1):196-7.

3. Sherman SL. Epidemiology. In: Hagerman R, Hagerman P, editors. Fragile X Syndrome: Diagnosis, Treatment and Research. Baltimore: The Johns Hopkins University Press; 2002. p. 136-68.

4. Terracciano A, Chiurazzi P, Neri G. Fragile X syndrome. Am J Med Genet C Semin Med Genet. 2005;137(1):32-7.

5. Oberle I, Rousseau F, Heitz D, Kretz C, Devys D, Hanauer A, et al. Instability of a 550-base pair DNA segment and abnormal methylation in fragile X syndrome. Science. 1991;252(5010):1097102.

6. Verkerk AJ, Pieretti M, Sutcliffe JS, Fu YH, KuhI DP, Pizzuti A, et al. Identification of a gene (FMR1) containing a CGG repeat coincident with a breakpoint cluster region exhibiting length variation in fragile $X$ syndrome. Cell. 1991;65(5):905-14.

7. Yu S, Pritchard M, Kremer E, Lynch M, Nancarrow J, Baker E, et al. Fragile X genotype characterized by an unstable region of DNA. Science. 1991;252(5010):1179-81.

8. Jin P, Alisch RS, Warren ST. RNA and microRNAs in fragile $\mathrm{X}$ mental retardation. Nat Cell Biol. 2004;6(11):1048-53.

9. Veltman JA, Brunner HG. De novo mutations in human genetic disease. Nat Rev Genet. 2012;13(8):565-75.

10. Exome Aggregation Consortium, Lek M, Karczewski K, Minikel E, Samocha K, Banks E, et al. Analysis of protein-coding genetic variation in 60,706 humans. Nature. 2016;536(7616):285-91.

11. Ripke S, O'Dushlaine C, Chambert K, Moran JL, Kähler A, Akterin S, et al. Genome-wide association analysis identifies 13 new risk loci for schizophrenia. Nature Genetics. 2013;45:11509.

12. Schizophrenia Working Group of the Psychiatric Genomics Consortium. Biological insights from 108 schizophrenia-associated genetic loci. Nature. 2014;511:421-7.

13. Altshuler DM, Gibbs RA, Peltonen L, Dermitzakis E, Schaffner SF, Yu F, et al. Integrating common and rare genetic variation in diverse human populations. Nature. 2010;467(7311):52-8.

14. Taylor AK, Safanda JF, Fall MZ, Quince C, Lang KA, Hull CE, et al. Molecular predictors of cognitive involvement in female carriers of fragile $X$ syndrome. Jama. 1994;271(7):507-14.

15. Merenstein SA, Sobesky WE, Taylor AK, Riddle JE, Tran HX, Hagerman RJ. Molecular-clinical correlations in males with an expanded FMR1 mutation. Am J Med Genet. 1996;64(2):388-94.

16. Loesch DZ, Huggins RM, Bui QM, Taylor AK, Hagerman RJ. Relationship of deficits of FMR1 gene specific protein with physical phenotype of fragile $X$ males and females in pedigrees: a new perspective. Am J Med Genet A. 2003;118(2):127-34. 
17. Chang CC, Chow CC, Tellier LC, Vattikuti S, Purcell SM, Lee JJ. Second-generation PLINK: rising to the challenge of larger and richer datasets. Gigascience. 2015;4:7.

18. Gerhardt J, Zaninovic N, Zhan Q, Madireddy A, Nolin SL, Ersalesi N, et al. Cis-acting DNA sequence at a replication origin promotes repeat expansion to fragile $X$ full mutation. J Cell Biol. 2014;206(5):599-607.

19. Barrett JC, Fry B, Maller J, Daly MJ. Haploview: analysis and visualization of LD and haplotype maps. Bioinformatics. 2005;21:263-5.

20. Price AL, Patterson NJ, Plenge RM, Wein blatt ME, Shadick NA, Reich D. Principal components analysis corrects for stratification in genome-wide association studies. Nat Genet.

2006;38(8):904-9.

21. Hindorff LA, Sethupathy P, Junkins HA, Ramos EM, Mehta JP, Collins FS, et al. Potential etiologic and functional implications of genome-wide association loci for human diseases and traits. Proc Natl Acad Sci U S A. 2009;106(23):9362-7.

22. Fromer M, Roussos P, Sieberts SK, Johnson JS, Kavanagh DH, Perumal T, et al. Gene expression elucidates functional impact of polygenic risk for schizophrenia. Nature Neuroscience. 2016;19(11):1442-53.

23. Peltonen L, Jalanko A, Varilo T. Molecular genetics of the Finnish disease heritage. Hum Mol Genet. 1999;8(10):1913-23.

24. Struewing JP, Hartge $P$, Wacholder S, Baker SM, Berlin M, McAdams M, et al. The risk of cancer associated with specific mutations of BRCA1 and BRCA2 among Ashkenazi Jews. N Engl J Med. 1997;336(20):1401-8.

25. Anderson CA, Soranzo N, Zeggini E, Barrett JC. Synthetic associations are unlikely to account for many common disease genome-wide association signals. PLoS Biol. 2011;9(1):e1000580.

26. Berg IL, Neumann R, Lam KW, Sarbajna S, Odenthal-Hesse L, May CA, et al. PRDM9 variation strongly influences recombination hot-spot activity and meiotic instability in humans. Nature genetics. 2010;42(10):859-63.

27. Hastings PJ, Lupski JR, Rosenberg SM, Ira G. Mechanisms of change in gene copy number. Nat Rev Genet. 2009;10(8):551-64.

28. Koren A, Polak P, Nemesh J, Michaelson JJ, Sebat J, Sunyaev SR, et al. Differential relationship of DNA replication timing to different forms of human mutation and variation. Am J Hum Genet. 2012;91(6):1033-40.

29. Libby RT, Hagerman KA, Pineda VV, Lau R, Cho DH, Baccam SL, et al. CTCF cis-regulates trinucleotide repeat instability in an epigenetic manner: a novel basis for mutational hot spot determination. PLoS Genet. 2008;4(11):e1000257.

30. Brock GJ, Anderson NH, Monckton DG. Cis-acting modifiers of expanded CAG/CTG triplet repeat expandability: associations with flanking $\mathrm{GC}$ content and proximity to $\mathrm{CpG}$ islands. Hum Mol Genet. 1999;8(6):1061-7. 
31. Eichler EE, Holden JJ, Popovich BW, Reiss AL, Snow K, Thibodeau SN, et al. Length of uninterrupted CGG repeats determines instability in the FMR1 gene. Nat Genet. 1994;8(1):88-

94.

32. Chiurazzi $P$, Macpherson J, Sherman $S$, Neri G. Significance of linkage disequilibrium between the fragile X locus and its flanking markers. Am J Med Genet. 1996;64(1):203-8.

33. Richards RI, Holman K, Friend K, Kremer E, Hillen D, Staples A, et al. Evidence of founder chromosomes in fragile X syndrome. Nat Genet. 1992;1(4):257-60.

34. Warby SC, Montpetit A, Hayden AR, Carroll JB, Butland SL, Visscher H, et al. CAG expansion in the Huntington disease gene is associated with a specific and targetable predisposing haplogroup. Am J Hum Genet. 2009;84(3):351-66. 


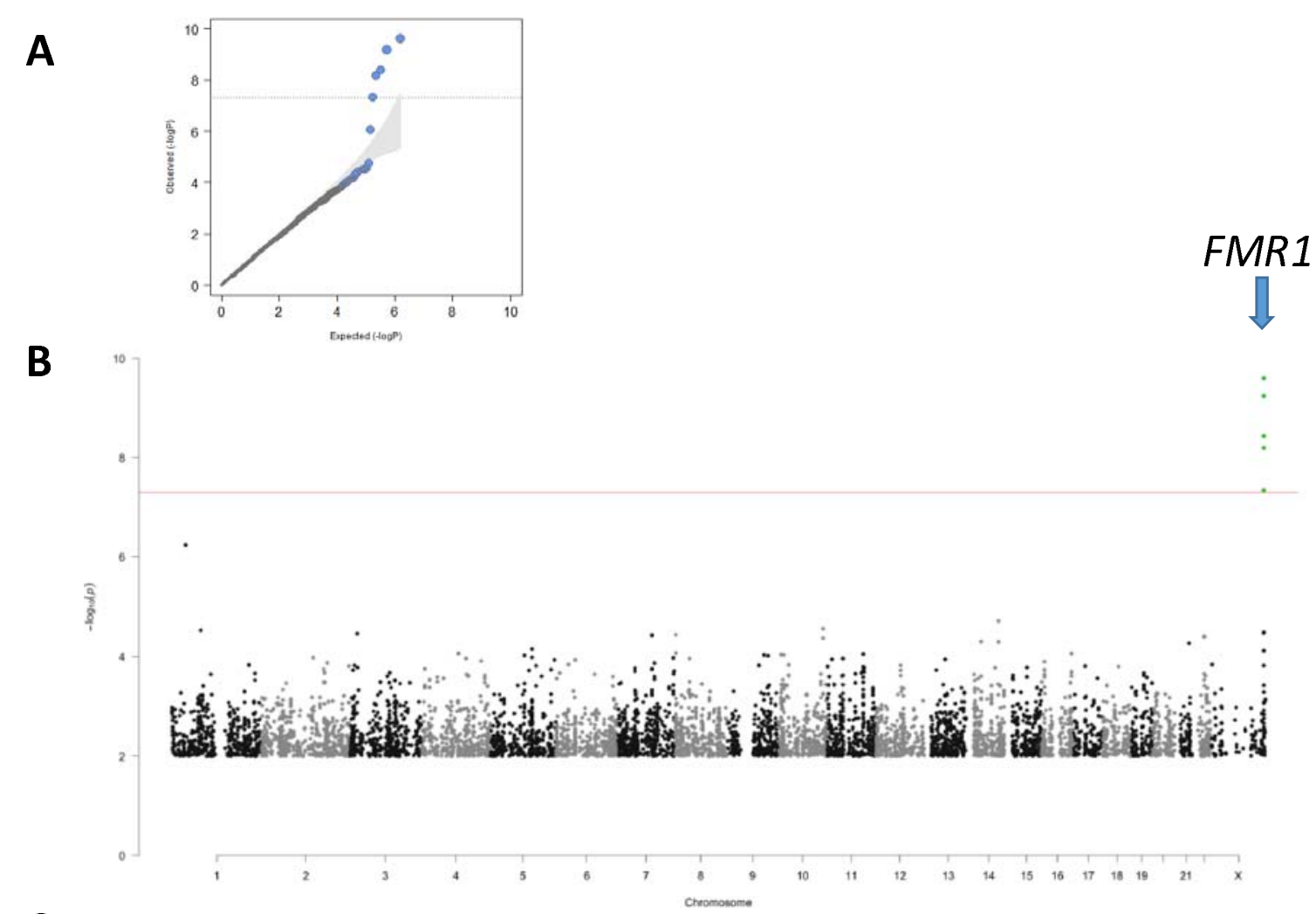

C

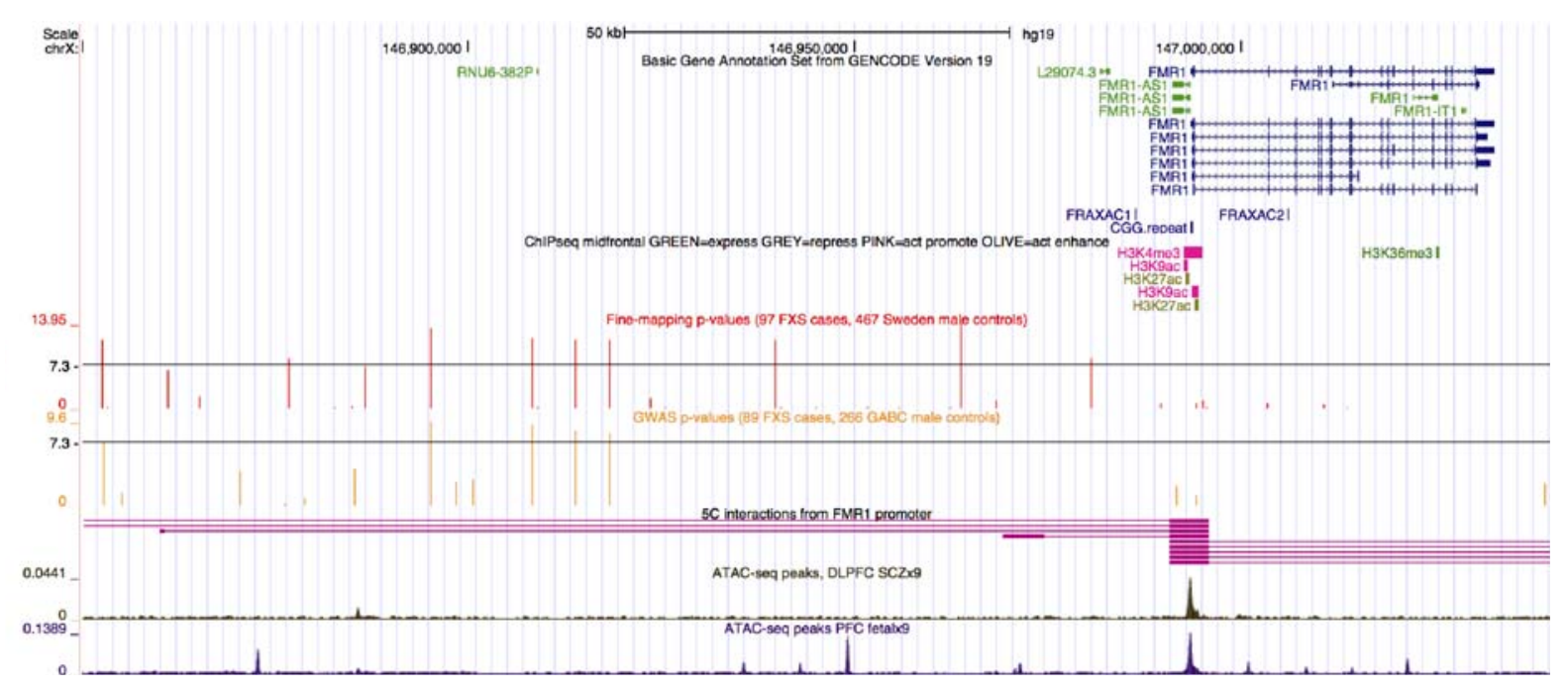

Figure 1. FXS case-control GWAS. (A) Quantile-quantile plot for logistic regression of male FXS cases and GABC controls (including ancestry principle components). The observed $P$-values conform closely to the null except for five SNPS in the FMR1 region. The shaded region indicates the expected 95\% probability interval for ordered P-values. (B) Manhattan plot for the GWAS of male FXS cases and GABC controls (logistic regression including ancestry principle components). The $X$-axis is chromosomal position from 1ptel to Xqtel. The $Y$-axis is - $\log _{10}(P)$. Genome-wide significant SNPS near FMR1 are indicated. (C) Detailed of FMR1 region (hg19, chrX:146850000-147040000). Tracks are: GENCODE gene annotations; positions of FRAXAC1, FRAXAC2, and promoter CGG repeat; selected ChIP-seq marks; SNP positions and - $\log _{10}(P)$ for SNPs in the fine-mapping study and in the GWAS; DNA-DNA chromosomal 
bioRxiv preprint doi: https://doi.org/10.1101/115998; this version posted March 11,2017 . The copyright holder for this preprint (which was not certified by peer review) is the author/funder. All rights reserved. No reuse allowed without permission.

looping from 5C based on the FMR1 promoter; and open chromatin in pre-frontal cortex of 9 adult SCZ cases and 9 fetal samples. 
Table 1. Summary of samples and genotyping.

\begin{tabular}{|c|c|c|c|c|}
\hline Purpose & Genotyping & FXS Cases & Controls & Control source \\
\hline Establish FXS status & $\begin{array}{l}\text { FMR1 5'UTR CGG repeat (CLIA assay) } \\
\text { FMR1 CGG repeat analysis }\end{array}$ & $\begin{array}{c}89 \\
82+\end{array}$ & $\begin{array}{c}0 \\
109\end{array}$ & $\begin{array}{c}\mathrm{N} / \mathrm{A} \\
\text { Sweden }\end{array}$ \\
\hline GWAS & Illumina HumanOmni1-Quad & 89 & 266 & GABC \\
\hline Allele frequency comparisons & $\begin{array}{l}\text { Illumina Human } 1 \mathrm{M} \& \text { Affymetrix } 6.0 \\
\text { Illumina OmniExpress }\end{array}$ & - & $\begin{array}{c}101 \\
3,525\end{array}$ & $\begin{array}{l}\text { HapMap3 } \\
\text { Sweden }\end{array}$ \\
\hline Verify key genotypes & $\begin{array}{l}\text { TaqMan rs2197706 } \\
\text { TaqMan rs5905149 }\end{array}$ & $\begin{array}{l}82+ \\
82+\end{array}$ & $\begin{array}{c}0 \\
94\end{array}$ & $\begin{array}{c}\text { N/A } \\
\text { Sweden }\end{array}$ \\
\hline Common variant fine-mapping & $\begin{array}{l}\text { Sequenom ( } 31 \text { SNPs) } \\
\text { TaqMan rs45631657 }\end{array}$ & $\begin{array}{c}97 \\
103\end{array}$ & $\begin{array}{l}467 \\
467\end{array}$ & $\begin{array}{l}\text { Sweden } \\
\text { Sweden }\end{array}$ \\
\hline
\end{tabular}

+ insufficient DNA for 7 FXS cases. 
Table 2. Genome-wide significant results of GWAS of male FXS cases and controls.

\begin{tabular}{|l|l|l|l|l|l|l|l|l|l|}
\hline SNP & chrX (hg19) & Alleles & OR (95\% Cl) & $P$ & $F_{\text {case }}$ & $F_{\text {control }}$ & $F_{\text {Sweden }}$ & $F_{\text {CEU }}$ & $F_{\text {TSI }}$ \\
\hline rs5952060 & 146852679 & C/T & $5.33(2.93-9.71)$ & $4.57 \times 10^{-8}$ & 0.764 & 0.365 & 0.357 & 0.386 & 0.409 \\
\hline rs2197706 & 146895120 & A/C & $8.10(4.24-15.49)$ & $2.53 \times 10^{-10}$ & 0.807 & 0.351 & - & 0.357 & 0.386 \\
\hline rs5905149 & 146908213 & A/C & $5.99(3.40-10.56)$ & $5.76 \times 10^{-10}$ & 0.584 & 0.184 & 0.183 & - & - \\
\hline rs7876251 & 146913828 & G/A & $5.64(3.17-10.01)$ & $3.68 \times 10^{-9}$ & 0.693 & 0.286 & 0.279 & 0.263 & 0.279 \\
\hline rs4824253 & 146918268 & G/A & $5.35(3.04-9.43)$ & $6.41 \times 10^{-9}$ & 0.685 & 0.286 & 0.278 & 0.263 & 0.296 \\
\hline
\end{tabular}

The first allele given is the least common in this sample and is the reference for the odds ratio (OR) and frequencies. $\mathrm{Cl}$ is confidence interval. $P$ is from the logistic regression including ancestry covariates. Logistic regression $P$-values after removing nine cases with divergent ancestry dropping were $5.86 \times 10^{-8}, 3.36 \times 10^{-10}, 8.52 \times 10^{-10}, 4.79 \times 10^{-9}$, and $8.38 \times 10^{-9}$ (respectively). Shown are allele frequencies for male FXS cases $\left(F_{\text {case }}\right)$, GABC controls $\left(F_{\text {control }}\right)$, subjects from Sweden $\left(F_{\text {Sweden }}\right)$, and HapMap3 northwestern European $\left(F_{C E U}\right)$ and Tuscan control samples $\left(F_{T S I}\right)$. 
Table 3. Haplotype analyses of FMR1 region.

\begin{tabular}{|l|c|c|c|c|c|}
\hline Haplotype & Subjects & Controls & FXS cases & Freq control & Freq case \\
\hline TGACGGTCC & 17 & 14 & 3 & 0.030 & 0.031 \\
\hline CGACGGTCC & 20 & 17 & 3 & 0.036 & 0.031 \\
\hline CGAAGGTTT & 21 & 20 & 1 & 0.043 & 0.010 \\
\hline CGACAATTC & 26 & 17 & 9 & 0.036 & 0.093 \\
\hline CGCCAATCC & 31 & 29 & 2 & 0.062 & 0.021 \\
\hline CGAAGGTTC & 65 & 42 & 23 & 0.090 & 0.237 \\
\hline CCAAGGCTT & 77 & 44 & 33 & 0.094 & 0.340 \\
\hline TGCCAATCC & 261 & 250 & 11 & 0.535 & 0.113 \\
\hline
\end{tabular}

Observed haplotypes from the fine-mapping data (32 SNPs in 97 FXS cases and 467 male controls). Haplotypes were created using nine genome-wide significant SNPs (rs5952060-rs112146098-rs2197706-rs5905149rs7876251-rs4824253-rs45631657 -rs4824231-rs25705). Haplotypes counts $<10$ ( $N=46)$ were removed. Logistic regression highlighted a strongly protective haplotypes (green) and two risk haplotypes (red). 